\title{
Higher-order duality in nondifferentiable minimax fractional programming involving generalized convexity
}

\section{Ahmad*}

"Correspondence:

drizhar@kfupm.edu.sa

Department of Mathematics and

Statistics, King Fahd University of Petroleum and Minerals, Dhahran,

31261, Saudi Arabia

Permanent address: Department of

Mathematics, Aligarh Muslim

University, Aligarh, 202 002, India

\begin{abstract}
A higher-order dual for a non-differentiable minimax fractional programming problem is formulated. Using the generalized higher-order $\eta$-convexity assumptions on the functions involved, weak, strong and strict converse duality theorems are established in order to relate the primal and dual problems. Results obtained in this paper naturally unify and extend some previously known results on non-differentiable minimax fractional programming in the literature.

MSC: 26A51; 90C32; 49N15
\end{abstract}

Keywords: fractional programming; nondifferentiable programming; higher-order duality; optimal solutions; generalized higher-order convexity

\section{Introduction}

The problem to be considered in the present analysis is the following nondifferentiable minimax fractional problem:

$$
\begin{gathered}
\text { Minimize } \sup _{y \in Y} \frac{f(x, y)+\left(x^{T} B x\right)^{1 / 2}}{g(x, y)-\left(x^{T} C x\right)^{1 / 2}}, \\
\text { subject to } h(x) \leq 0, x \in R^{n},
\end{gathered}
$$

where $Y$ is a compact subset of $R^{l}, f(\cdot, \cdot), g(\cdot, \cdot): R^{n} \times R^{l} \rightarrow R$, and $h(\cdot): R^{n} \rightarrow R^{m}$ are continuously differentiable functions. $B$ and $C$ are $n \times n$ positive semidefinite symmetric matrices. It is assumed that for each $(x, y)$ in $R^{n} \times R^{l}, f(x, y)+\left(x^{T} B x\right)^{\frac{1}{2}} \geq 0$ and $g(x, y)-\left(x^{T} C x\right)^{\frac{1}{2}}>0$.

In an earlier work, Schmittendorf [1] established necessary and sufficient optimality conditions for the following minimax programming problem:

Minimize $\sup _{y \in Y} f(x, y)$, subject to $h(x) \leq 0, x \in R^{n}$,

where $Y$ is a compact subset of $R^{l}$, the functions $f(\cdot, \cdot): R^{n} \times R^{l} \rightarrow R$, and $h(\cdot): R^{n} \rightarrow R^{m}$ are in $C^{1}$.

( 2012 Ahmad; licensee Springer. This is an Open Access article distributed under the terms of the Creative Commons Attribution License (http://creativecommons.org/licenses/by/2.0), which permits unrestricted use, distribution, and reproduction in any medium, provided the original work is properly cited. 
Tanimoto [2] applied the necessary conditions in [1] to formulate a dual problem and discussed the duality results, which were extended to fractional analogue of $\left(\mathrm{P}_{1}\right)$ by several authors [3-12]. Liu [13] proposed the second-order duality theorems for $\left(\mathrm{P}_{1}\right)$ under generalized second-order B-invex functions. Mishra and Rueda [14] and Ahmad, Husain and Sarita [15] discussed the second-order duality results for the following nondifferentiable minimax programming problems:

$$
\text { Minimize } \sup _{y \in Y} f(x, y)+\left(x^{t} B x\right)^{1 / 2} \text {, }
$$

subject to $h(x) \leq 0, x \in R^{n}$,

where $Y$ is a compact subset of $R^{l}, f(\cdot, \cdot): R^{n} \times R^{l} \rightarrow R$, and $h(\cdot): R^{n} \rightarrow R^{m}$ are twice differentiable functions. $B$ is an $n \times n$ positive semidefinite symmetric matrix. Ahmad, Husain and Sharma [16] formulated a unified higher-order dual of $\left(\mathrm{P}_{2}\right)$ and established weak, strong and strict converse duality theorems under higher-order $(F, \alpha, \rho, d)$-Type I assumptions. Recently, Jayswal and Stancu-Minasian [17] obtained higher-order duality results for $\left(\mathrm{P}_{2}\right)$.

In this paper, we formulate a higher-order dual of $(\mathrm{P})$ and establish weak, strong and strict converse duality theorems under generalized higher-order $\eta$-convexity assumptions. More precisely, this paper is an extension of second-order duality results of $\mathrm{Hu}$, Chen and Jian [18] to a class of higher-order duality and it also presents an answer to a question raised in [17].

\section{Notation and preliminaries}

Let $\mathcal{X}=\left\{x \in R^{n}: h(x) \leq 0\right\}$ denote the set of all feasible solutions of (NFP). Any point $x \in \mathcal{X}$ is called a feasible point of (NFP). For each $(x, y) \in R^{n} \times R^{l}$, we define

$$
\psi(x, y)=\frac{f(x, y)+\left(x^{T} B x\right)^{1 / 2}}{g(x, y)-\left(x^{T} C x\right)^{1 / 2}}
$$

such that for each $(x, y) \in \mathcal{X} \times Y$,

$$
f(x, y)+\left(x^{T} B x\right)^{1 / 2} \geq 0 \text { and } g(x, y)-\left(x^{T} C x\right)^{1 / 2}>0 .
$$

For each $x \in \mathcal{X}$, we define

$$
J(x)=\left\{j \in J: h_{j}(x)=0\right\}
$$

where

$$
\begin{aligned}
& J=\{1,2, \ldots, m\}, \\
& Y(x)=\left\{y \in Y: \frac{f(x, y)+\left(x^{T} B x\right)^{1 / 2}}{g(x, y)-\left(x^{T} C x\right)^{1 / 2}}=\sup _{z \in Y} \frac{f(x, z)+\left(x^{T} B x\right)^{1 / 2}}{g(x, z)-\left(x^{T} C x\right)^{1 / 2}}\right\} . \\
& S(x)=\left\{(s, t, \tilde{y}) \in N \times R_{+}^{s} \times R^{l s}: 1 \leq s \leq n+1, t=\left(t_{1}, t_{2}, \ldots, t_{s}\right) \in R_{+}^{s}\right.
\end{aligned}
$$




$$
\text { with } \left.\sum_{i=1}^{s} t_{i}=1, \tilde{y}=\left(\bar{y}_{1}, \bar{y}_{2}, \ldots, \bar{y}_{s}\right) \text { with } \bar{y}_{i} \in Y(x)(i=1,2, \ldots, s)\right\} \text {. }
$$

Since $f$ and $g$ are continuously differentiable and $Y$ is compact in $R^{l}$, it follows that for each $x^{*} \in \mathcal{X}, Y\left(x^{*}\right) \neq \emptyset$, and for any $\bar{y}_{i} \in Y\left(x^{*}\right)$, we have a positive constant

$$
\lambda_{\circ}=\psi\left(x^{*}, \bar{y}_{i}\right)=\frac{f\left(x^{*}, \bar{y}_{i}\right)+\left(x^{* T} B x^{*}\right)^{1 / 2}}{g\left(x^{*}, \bar{y}_{i}\right)-\left(x^{* T} C x^{*}\right)^{1 / 2}} .
$$

Lemma 2.1 (Generalized Schwarz inequality) Let $A$ be a positive-semidefinite matrix of order $n$. Then, for all $x, w \in R^{n}$,

$$
x^{T} A w \leq\left(x^{T} A x\right)^{\frac{1}{2}}\left(w^{T} A w\right)^{\frac{1}{2}} .
$$

The equality $A x=\xi A w$ holds for some $\xi \geq 0$. Clearly, if $\left(w^{T} A w\right)^{\frac{1}{2}} \leq 1$, we have

$$
x^{T} A w \leq\left(x^{T} A x\right)^{\frac{1}{2}} .
$$

We will use the following definitions.

Let $\phi: R^{n} \rightarrow R$ and $k: R^{n} \times R^{n} \rightarrow R$ be differentiable functions.

Definition 2.1 [19] A function $\phi$ is said to be higher-order $\eta$-convex if there exists a certain mapping $\eta: R^{n} \times R^{n} \rightarrow R^{n}$ such that for all $x, p \in R^{n}$, we have

$$
\phi(x)-\phi(\bar{x})-k(\bar{x}, p)+p^{T} \nabla_{p} k(\bar{x}, p) \geq \eta(x, \bar{x})^{T} \nabla_{p} k(\bar{x}, p) .
$$

Definition 2.2 [19] A function $\phi$ is said to be higher-order (strictly) $\eta$-pseudoconvex if there exists a certain mapping $\eta: R^{n} \times R^{n} \rightarrow R^{n}$ such that for all $x, p \in R^{n}$, we have

$$
\begin{aligned}
& \eta^{T}(x, \bar{x}) \nabla_{p} k(\bar{x}, p) \geq 0 \\
& \quad \Rightarrow \quad \phi(x)(>) \geq \phi(\bar{x})+k(\bar{x}, p)-p^{T} \nabla_{p} k(\bar{x}, p) .
\end{aligned}
$$

Definition 2.3 [19] A function $\phi$ is said to be higher-order (strictly) $\eta$-quasiconvex if there exists a certain mapping $\eta: R^{n} \times R^{n} \rightarrow R^{n}$ such that for all $x, p \in R^{n}$, we have

$$
\begin{aligned}
& \eta^{T}(x, \bar{x}) \nabla_{p} k(\bar{x}, p) \geq 0 \\
& \quad \Rightarrow \quad \phi(x) \leq \phi(\bar{x})+k(\bar{x}, p)-p^{T} \nabla_{p} k(\bar{x}, p)(<) \leq 0 .
\end{aligned}
$$

\section{Higher-order duality model}

In this section, we formulate the higher-order dual for (NFP) and derive duality results.

$$
\max _{(s, t, \tilde{y}) \in S(z)} \sup _{(z, \mu, \lambda, v, w, p) \in L(s, t, \tilde{y})} \lambda,
$$

where $L(s, t, \tilde{y})$ denotes the set of all $(z, \mu, \lambda, v, w, p) \in R^{n} \times R_{+}^{m} \times R_{+} \times R^{n} \times R^{n} \times R^{n}$ subject to

$$
\sum_{i=1}^{s} t_{i}\left[\nabla_{p}\left(F\left(z, \bar{y}_{i}, p\right)-\lambda G\left(z, \bar{y}_{i}, p\right)\right)\right]+B w+\lambda C v+\sum_{j=1}^{m} \mu_{j} \nabla_{p} H_{j}(z, p)=0,
$$




$$
\begin{aligned}
& \sum_{i=1}^{s} t_{i}\left[f\left(z, \bar{y}_{i}\right)+z^{T} B w-\lambda\left(g\left(z, \bar{y}_{i}\right)-z^{T} C v\right)+F\left(z, \bar{y}_{i}, p\right)-\lambda G\left(z, \bar{y}_{i}, p\right)+\sum_{j \in J_{0}} \mu_{j} h_{j}(z)\right. \\
& \quad-p^{T} \nabla_{p}\left\{F\left(z, \bar{y}_{i}, p\right)-\lambda G\left(z, \bar{y}_{i}, p\right)\right\} \\
& \left.\quad+\sum_{j \in J_{0}} \mu_{j} H_{j}(z, p)-p^{T} \sum_{j \in J_{0}} \mu_{j} \nabla_{p} H_{j}(z, p)\right] \geq 0, \\
& \sum_{j \in J_{\alpha}} \mu_{j}\left[h_{j}(z)+H_{j}(z, p)-p^{T} \nabla_{p} H_{j}(z, p)\right] \geq 0, \quad \alpha=1,2, \ldots, r, \\
& w^{T} B w \leq 1, \quad v^{T} C v \leq 1, \quad\left(z^{T} B z\right)^{\frac{1}{2}}=z^{T} B w, \quad\left(z^{T} C z\right)^{\frac{1}{2}}=z^{T} C v,
\end{aligned}
$$

where $J_{\alpha} \subseteq M=\{1,2, \ldots, m\}, \alpha=0,1,2, \ldots, r$ with $\bigcup_{\alpha=0}^{r} J_{\alpha}=M$ and $J_{\alpha} \cap J \beta=\emptyset$ if $\alpha \neq \beta$. If for a triplet $(s, t, \tilde{y}) \in S(z)$, the set $L(s, t, \tilde{y})=\varnothing$, then we define the supremum over it to be $\infty$.

Remark 3.1 (i) Let $J_{0}=\emptyset, F\left(z, \bar{y}_{i}, p\right)=p^{T} \nabla f\left(z, \bar{y}_{i}\right)+\frac{1}{2} p^{T} \nabla^{2} f\left(z, \bar{y}_{i}\right) p, G\left(z, \bar{y}_{i}, p\right)=p^{T} \nabla$ $g\left(z, \bar{y}_{i}\right)+\frac{1}{2} p^{T} \nabla^{2} g\left(z, \bar{y}_{i}\right) p, i=1,2, \ldots, s$ and $H_{j}(z, p)=p^{T} \nabla h_{j}(z)+\frac{1}{2} p^{T} \nabla^{2} h_{j}(z) p, j=1,2, \ldots, m$. Then (NMD) reduces to the second-order dual in $[18,20]$. If, in addition, $p=0$, then we get the dual formulated by Ahmad, Gupta, Kailey and Agarwal [5].

(ii) If $J_{0}=\emptyset$, then the above dual becomes the dual formulated in [21].

Theorem 3.1 (Weak duality) Let $x$ and $(z, \mu, \lambda, s, t, v, w, \tilde{y}, p)$ be feasible solutions of (NFP) and (NMD) respectively. Assume that

(i) $\left[\sum_{i=1}^{s} t_{i}\left\{f\left(\cdot, \bar{y}_{i}\right)+(\cdot)^{T} B w-\lambda\left(g\left(\cdot, \bar{y}_{i}\right)-(\cdot)^{T} C v\right)\right\}+\sum_{j \in J_{0}} \mu_{j} h_{j}(\cdot)\right]$ is higher-order $\eta$-pseudoconvex at $z$,

(ii) $\sum_{j \in J_{\alpha}} \mu_{j} h_{j}(\cdot), \alpha=1,2, \ldots, r$, is higher-order $\eta$-quasiconvex at $z$.

Then

$$
\sup _{y \in Y} \frac{f(x, y)+\left(x^{t} B x\right)^{1 / 2}}{g(x, y)-\left(x^{t} C x\right)^{1 / 2}} \geq \lambda .
$$

Proof Suppose to the contrary that

$$
\sup _{y \in Y} \frac{f(x, y)+\left(x^{T} B x\right)^{1 / 2}}{g(x, y)-\left(x^{T} C x\right)^{1 / 2}}<\lambda .
$$

Then we have

$$
f\left(x, \bar{y}_{i}\right)+\left(x^{T} B x\right)^{1 / 2}-\lambda\left(g\left(x, \bar{y}_{i}\right)-\left(x^{T} C x\right)^{1 / 2}\right)<0, \quad \text { for all } \bar{y}_{i} \in Y, i=1,2, \ldots, s .
$$

It follows from $t_{i} \geq 0, i=1,2, \ldots, s$, that

$$
t_{i}\left[f\left(x, \bar{y}_{i}\right)+\left(x^{T} B x\right)^{1 / 2}-\lambda\left(g\left(x, \bar{y}_{i}\right)-\left(x^{T} C x\right)^{1 / 2}\right)\right] \leq 0, \quad i=1,2, \ldots, s,
$$

with at least one strict inequality since $t=\left(t_{1}, t_{2}, \ldots, t_{s}\right) \neq 0$. Taking summation over $i$ and using $\sum_{i=1}^{s} t_{i}=1$, we have

$$
\sum_{i=1}^{s} t_{i}\left[f\left(x, \bar{y}_{i}\right)+\left(x^{T} B x\right)^{1 / 2}-\lambda\left(g\left(x, \bar{y}_{i}\right)-\left(x^{T} C x\right)^{1 / 2}\right)\right]<0 .
$$


It follows from the generalized Schwarz inequality and (3.4) that

$$
\sum_{i=1}^{s} t_{i}\left[f\left(x, \bar{y}_{i}\right)+x^{T} B w-\lambda\left(g\left(x, \bar{y}_{i}\right)-x^{T} C v\right)\right]<0 .
$$

By the feasibility of $x$ for (NFP)and $\mu \geq 0$, we obtain

$$
\sum_{j \in J_{0}} \mu_{j} h_{j}(x) \leq 0
$$

The above inequality with (3.5) gives

$$
\sum_{i=1}^{s} t_{i}\left[f\left(x, \bar{y}_{i}\right)+x^{T} B w-\lambda\left(g\left(x, \bar{y}_{i}\right)-x^{T} C v\right)\right]+\sum_{j \in J_{0}} \mu_{j} h_{j}(x)<0 .
$$

Now, the feasibility of $x$ for (NFP), $\mu \geq 0$ and (3.3) yields

$$
\sum_{j \in J_{\alpha}} \mu_{j} h_{j}(x) \leq 0 \leq \sum_{j \in J_{\alpha}} \mu_{j}\left[h_{j}(z)+H_{j}(z, p)-p^{T} \nabla_{p} H_{j}(z, p)\right], \quad \alpha=1,2, \ldots, r .
$$

The inequality (3.8) and Hypothesis (ii) gives

$$
\eta^{T}(x, z)\left[\sum_{j \in J_{\alpha}} \mu_{j} \nabla_{p} H_{j}(z, p)\right] \leq 0, \quad \alpha=1,2, \ldots, r
$$

From (3.1) and (3.9), we have

$$
\begin{aligned}
& \eta^{T}(x, z)\left[\sum_{i=1}^{s} t_{i}\left\{\nabla_{p}\left(F\left(z, \bar{y}_{i}, p\right)+B w-\lambda\left(G\left(z, \bar{y}_{i}, p\right)-C v\right)\right)\right\}+\sum_{j \in J_{0}} \mu_{j} \nabla_{p} H_{j}(z, p)\right] \\
& \quad \geq 0,
\end{aligned}
$$

which by virtue of Hypothesis (i) yields

$$
\begin{aligned}
& \sum_{i=1}^{s} t_{i}\left[f\left(x, \bar{y}_{i}\right)+x^{T} B w-\lambda\left(g\left(x, \bar{y}_{i}\right)-x^{T} C v\right)\right]+\sum_{j \in J_{0}} \mu_{j} h_{j}(x) \\
& \geq \sum_{i=1}^{s} t_{i}\left[f\left(z, \bar{y}_{i}\right)+z^{T} B w-\lambda\left(g\left(z, \bar{y}_{i}\right)-z^{T} C v\right)\right. \\
& \quad+\sum_{j \in J_{0}} \mu_{j} h_{j}(z)+F\left(z, \bar{y}_{i}, p\right)-\lambda G\left(z, \bar{y}_{i}, p\right) \\
& \left.\quad-p^{T} \nabla_{p}\left\{F\left(z, \bar{y}_{i}, p\right)-\lambda G\left(z, \bar{y}_{i}, p\right)\right\}\right]+\sum_{j \in J_{0}} \mu_{j} H_{j}(z, p)-p^{T} \sum_{j \in J_{0}} \mu_{j} \nabla_{p} H_{j}(z, p) \\
& \geq 0 \quad(\text { by }(3.2)) .
\end{aligned}
$$

This contradicts (3.7). 
Theorem 3.2 (Strong duality) Let $x^{*}$ be an optimal solution of (NFP) and let $\nabla h_{j}\left(x^{*}\right), j \in$ $J\left(x^{*}\right)$ be linearly independent. Assume that

$$
\begin{array}{ll}
F\left(x^{*}, \bar{y}_{i}^{*}, 0\right)=0 ; & \nabla_{p} F\left(x^{*}, \bar{y}_{i}^{*}, 0\right)=\nabla f\left(x^{*}, \bar{y}_{i}^{*}\right), \quad i=1,2, \ldots, s, \\
G\left(x^{*}, \bar{y}_{i}^{*}, 0\right)=0 ; & \nabla_{p} G\left(x^{*}, \bar{y}_{i}^{*}, 0\right)=\nabla g\left(x^{*}, \bar{y}_{i}^{*}\right), \quad i=1,2, \ldots, s, \\
H_{j}\left(x^{*}, 0\right)=0 ; & \nabla_{p} H_{j}\left(x^{* \prime}, 0\right)=\nabla h_{j}\left(x^{*}\right), \quad j \in J .
\end{array}
$$

Then there exist $\left(s^{\prime \prime}, t^{*}, \tilde{y}^{\prime \prime}\right) \in S$ and $\left(x^{*}, \mu^{\prime \prime}, \lambda^{*}, v^{*}, w^{\prime \prime}, p^{*}\right) \in L\left(s^{*}, t^{*}, \tilde{y}^{*}\right)$ such that $\left(x^{*}, \mu^{*}, \lambda^{*}, v^{*}\right.$, $\left.w^{*}, s^{*}, t^{*}, \tilde{y}^{*}, p^{*}=0\right)$ is a feasible solution of (NMD) and the two objectives have the same values. Furthermore, if the assumptions of weak duality (Theorem 3.1) hold for all feasible solutions of (NFP) and (NMD), then $\left(x^{*}, \mu^{*}, \lambda^{*}, v^{*}, w^{*}, s^{*}, t^{*}, \tilde{y}^{*}, p^{*}=0\right)$ is an optimal solution of (NMD).

Proof Since $x^{*}$ is an optimal solution of (NFP) and $\nabla h_{j}\left(x^{*}\right), j \in J\left(x^{*}\right)$ are linearly independent, by the necessary conditions obtained in [13], there exist $\left(s^{*}, t^{*}, \tilde{y}^{*}\right) \in S$ and $\left(x^{*}, \mu^{*}, \lambda^{*}, v^{*}, w^{*}, p^{*}\right) \in L\left(s^{*}, t^{*}, \tilde{y}^{*}\right)$ such that $\left(x^{*}, \mu^{*}, \lambda^{*}, v^{*}, w^{*}, s^{*}, t^{*}, \tilde{y}^{*}, p^{*}=0\right)$ is a feasible solution of (NMD) and the problems (NFP) and (NMD) have the same objectives values and

$$
\lambda^{*}=\frac{f\left(x^{*}, \bar{y}_{i}^{*}\right)+\left(x^{* T} B x^{*}\right)^{1 / 2}}{g\left(x^{*}, \bar{y}_{i}^{*}\right)-\left(x^{* T} C x^{*}\right)^{1 / 2}} .
$$

Theorem 3.3 (Strict converse duality) Let $x^{\prime \prime}$ and $\left(z^{\prime \prime}, \mu^{\prime \prime}, \lambda^{\prime \prime}, s^{\prime \prime}, t^{\prime \prime}, v^{\prime \prime}, w^{\prime \prime}, \tilde{y}^{\prime \prime}, p^{*}\right)$ be the optimal solutions of (NFP) and (NMD), respectively. Assume that

(i) $\nabla h_{j}\left(x^{*}\right), j \in J\left(x^{*}\right)$ are linearly independent,

(ii) $\left[\sum_{i=1}^{s} t_{i}^{\prime \prime}\left\{f\left(\cdot, \bar{y}_{i}^{*}\right)+(\cdot)^{T} B w^{*}-\lambda^{\prime \prime}\left(g\left(\cdot, \bar{y}_{i}^{*}\right)-(\cdot)^{T} C v^{*}\right)\right\}+\sum_{j \in J_{0}} \mu_{j}^{* \prime} h_{j}(\cdot)\right]$ is higher-order strictly $\eta$-pseudoconvex at $z^{\prime \prime}$,

(iii) $\sum_{j \in J_{\alpha}} \mu_{j}^{\prime \prime} h_{j}(\cdot), \alpha=1,2, \ldots, r$, is higher-order $\eta$-quasiconvex at $z^{*}$.

Then, $z^{*}=x^{*}$; that is, $z^{*}$ is an optimal solution of (NFP).

Proof We will assume that $z^{\prime \prime} \neq x^{\prime \prime}$ and reach a contradiction. From the strong duality theorem (Theorem 3.2), it follows that

$$
\sup _{y \in Y} \frac{f\left(x^{*}, \tilde{y}^{*}\right)+\left(x^{*} T B x^{*}\right)^{1 / 2}}{g\left(x^{*}, \tilde{y}^{*}\right)-\left(x^{*} T C x^{*}\right)^{1 / 2}}=\lambda^{*} .
$$

Now, proceeding as in Theorem 3.1, we get

$$
\sum_{i=1}^{s} t_{i}^{*}\left[f\left(x^{*}, \bar{y}_{i}^{*}\right)+x^{*} T B w^{*}-\lambda^{*}\left(g\left(x^{*}, \bar{y}_{i}^{*}\right)-x^{*} T C v^{*}\right)\right]+\sum_{j \in J_{0}} \mu_{j}^{*} h_{j}\left(x^{*}\right)<0
$$

and

$$
\begin{aligned}
& \eta^{T}\left(x^{*}, z^{*}\right)\left[\sum_{i=1}^{s} t_{i}^{*}\left\{\nabla_{p}\left(F\left(z^{*}, \bar{y}_{i}^{*}, p^{*}\right)-\lambda^{*} G\left(z^{*}, \bar{y}_{i}^{*}, p^{*}\right)\right)+B w^{*}+\lambda^{*} C v^{*}\right\}\right. \\
& \left.\quad+\sum_{j \in J_{0}} \mu_{j}^{*} \nabla_{p} H_{j}\left(z^{*}, p^{*}\right)\right] \geq 0 .
\end{aligned}
$$


By Hypothesis (ii), (3.2) and (3.13), we have

$$
\sum_{i=1}^{s} t_{i}^{*}\left[f\left(x^{*}, \bar{y}_{i}^{*}\right)+x^{*} T B w^{*}-\lambda^{*}\left(g\left(x^{*}, \bar{y}_{i}^{*}\right)-x^{*} T C v^{*}\right)\right]+\sum_{j \in J_{0}} \mu_{j}^{*} h_{j}\left(x^{*}\right)>0,
$$

which contradicts (3.12). Hence the results.

\section{Concluding remarks}

The notion of higher-order invexity is adopted, which includes many other generalized convexity concepts in mathematical programming as special cases. If we take $J_{0}=\emptyset$, $F\left(z, \bar{y}_{i}, p\right)=p^{T} \nabla f\left(z, \bar{y}_{i}\right)+\frac{1}{2} p^{T} \nabla^{2} f\left(z, \bar{y}_{i}\right) p, G\left(z, \bar{y}_{i}, p\right)=p^{T} \nabla g\left(z, \bar{y}_{i}\right)+\frac{1}{2} p^{T} \nabla^{2} g\left(z, \bar{y}_{i}\right) p$, $i=1,2, \ldots, s$ and $H_{j}(z, p)=p^{T} \nabla h_{j}(z)+\frac{1}{2} p^{T} \nabla^{2} h_{j}(z) p, j=1,2, \ldots, m$ in Theorems 3.1-3.3, then we get Theorems 3.1-3.3 in [18].

The presented results in this paper can be further extended to the following related class of nondifferentiable minimax fractional programming problems:

$$
\begin{array}{r}
\operatorname{Min} \sup _{v \in W} \frac{\operatorname{Re}\left[\phi(\xi, v)+\left(z^{H} B z\right)^{1 / 2}\right]}{\operatorname{Re}\left[\psi(\xi, v)-\left(z^{H} D z\right)^{1 / 2}\right]} \\
\text { subject to }-g(\xi) \in S^{\circ}, \xi \in C^{2 n},
\end{array}
$$

where $\xi=(z, \bar{z}), v=(\omega, \bar{\omega})$ for $z \in C^{n}, \omega \in C^{l} . \phi(\cdot, \cdot): C^{2 n} \times C^{2 l} \rightarrow C$ and $\psi(\cdot, \cdot): C^{2 n} \times$ $C^{2 l} \rightarrow C$ are analytic with respect to $\xi, W$ is a specified compact subset in $C^{2 l}, S^{\circ}$ is a polyhedral cone in $C^{m}$ and $g: C^{2 n} \rightarrow C^{m}$ is analytic. Also $B, D \in C^{n \times n}$ are positive semidefinite Hermitian matrices.

\section{Competing interests}

The author declares that he has no competing interest.

\section{Acknowledgements}

The research of the author is supported by the Internal Project No. IN111015 of King Fahd University of Petroleum and Minerals, Dhahran-31261, Saudi Arabia.

Received: 4 September 2012 Accepted: 2 December 2012 Published: 20 December 2012

\section{References}

1. Schmitendorff, WE: Necessary conditions and sufficient conditions for static minimax problems. J. Math. Anal. Appl. 57, 683-693 (1977)

2. Tanimoto, S: Duality for a class of nondifferentiable mathematical programming problems. J. Math. Anal. Appl. 79, 283-294 (1981)

3. Ahmad, I, Husain, Z: Optimality conditions and duality in nondifferentiable minimax fractional programming with generalized convexity. J. Optim. Theory Appl. 129, 255-275 (2006)

4. Ahmad, I, Husain, Z: Duality in nondifferentiable minimax fractional programming with generalized convexity. Appl. Math. Comput. 176, 545-551 (2006)

5. Ahmad, I, Gupta, SK, Kailey, N, Agarwal, RP: Duality in nondifferentiable minimax fractional programming with $B$ - (p, r)-invexity. J. Inequal. Appl. 2011, 75 (2011)

6. Chandra, S, Kumar, V: Duality in fractional minimax programming. J. Aust. Math. Soc. A 58, 376-386 (1995)

7. Husain, Z, Ahmad, I, Sharma, S: Second order duality for minimax fractional programming. Optim. Lett. 3, 277-286 (2009)

8. Lai, HC, Liu, JC, Tanaka, K: Necessary and sufficient conditions for minimax fractional programming. J. Math. Anal. Appl. 230, 311-328 (1999)

9. Liu, JC, Wu, CS: On minimax fractional optimality conditions and invexity. J. Math. Anal. Appl. 219, 21-35 (1998)

10. Liu, JC, Wu, CS: On minimax fractional optimality conditions with $(\mathcal{F}, \rho)$-convexity. J. Math. Anal. Appl. 219, 36-51 (1998)

11. Yadav, SR, Mukherjee, RN: Duality in fractional minimax programming problems. J. Aust. Math. Soc. Series B, Appl. Math 31, 484-492 (1990)

12. Yang, XM, Hou, SH: On minimax fractional optimality conditions and duality with generalized convexity. J. Glob. Optim. 31, 235-252 (2005) 
13. Liu, JC: Second-order duality for minimax programming. Util. Math. 56, 53-63 (1999)

14. Mishra, SK, Rueda, NG: Second order duality for nondifferentiable minimax programming involving generalized type I functions. J. Optim. Theory Appl. 130, 479-488 (2006)

15. Ahmad, I, Husain, Z, Sharma, S: Second-order duality in nondifferentiable minimax programming involving type-I functions. J. Comput. Appl. Math. 215, 91-102 (2008)

16. Ahmad, I, Husain, Z, Sharma, S: Higher-order duality in nondifferentiable minimax programming with generalized type I functions. J. Optim. Theory Appl. 141, 1-12 (2009)

17. Jayswal, A, Stancu-Minasian, I: Higher-order duality in nondifferentiable minimax programming problem with generalized convexity. Nonlinear Anal., Theory Methods Appl. 74, 616-625 (2011)

18. Hu, Q, Chen, Y, Jian, J: Second-order duality for nondifferentiable minimax fractional programming. Int. J. Comput. Math. 89, 11-16 (2012)

19. Zhang, J: Generalized convexity and higher order duality for mathematical programming. Ph.D. Thesis, La Trobe University, Australia (1998)

20. Ahmad, I: Second order nondifferentiable minimax fractional programming with square root terms. Filomat 27, 126-133 (2013)

21. Jin, C, Ceng, CZ: Higher-order duality for minimax fractional type programming involving symmetric matrices. Appl. Math. 2, 1387-1392 (2011)

doi:10.1186/1029-242X-2012-306

Cite this article as: Ahmad: Higher-order duality in nondifferentiable minimax fractional programming involving generalized convexity. Journal of Inequalities and Applications 2012 2012:306.

\section{Submit your manuscript to a SpringerOpen ${ }^{\circ}$ journal and benefit from:}

- Convenient online submission

- Rigorous peer review

- Immediate publication on acceptance

- Open access: articles freely available online

- High visibility within the field

- Retaining the copyright to your article 